Corrigendum

\title{
Duplication of the inferior vena cava associated with other variations
}

Hao-Gang Xue, Chun-Ying Yang, Mitsuo Asakawa, Kumiko Tanuma and Hitoshi Ozawa

Anatomical Science International (2007) 82, 121-125

There was a typographical error in the catchline. It was printed as Anatomical Science International (2006) 82, 121-125 but the year should have been 2007.

The publishers apologize for this error. 